INPLASY

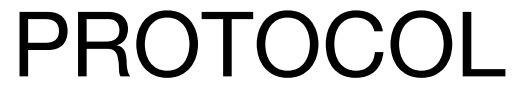

To cite: Lacerda et al.

Accuracy of ultrasonography in breast implants rupture diagnostic: systematic review and meta-analysis. Inplasy protocol 202060104. doi: 10.37766/inplasy2020.6.0104

Received: 27 June 2020

Published: 27 June 2020

Corresponding author: George da Silva Carvalho

gescarvalho@gmail.com

Author Affiliation:

Universidade do Extremo Sul Catarinense

Support: Not applicable.

Review Stage at time of this submission: Data extraction.

Conflicts of interest:

The present study has no conflict of interest.

\section{Accuracy of ultrasonography in breast implants rupture diagnostic: systematic review and meta-analysis}

Lacerda, ACM푸 Uggioni, MLR2; Carvalho, GS33 Simon, CS4; Cruz, M5; Silva, F6; Rosa, Ml7.

Review question / Objective: P- Population: Women with mammary prostheses; I- Index test- Ultrasound of breasts; $\mathrm{C}$ Comparison: Breast surgery findings; O- Outcome - Breast implant rupture.

Condition being studied: The aim of this systematic review is to define if an ultrasound has a satisfactory accuracy in the screening of breast implant ruptures, beside the fact that it is an accessible tool.

Information sources: MEDLINE, EMBASE, Cochrane Library, Insight and Grey literature (Google Scholar and the British Library)

INPLASY registration number: This protocol was registered with the International Platform of Registered Systematic Review and Meta-Analysis Protocols (INPLASY) on 27 June 2020 and was last updated on 27 June 2020 (registration number INPLASY202060104).

\section{INTRODUCTION}

Review question / Objective: P- Population: Women with mammary prostheses; I- Index test- UItrasound of breasts; C-
Comparison: Breast surgery findings; 0 Outcome - Breast implant rupture.

Condition being studied: The aim of this systematic review is to define if an ultrasound has a satisfactory accuracy in 
the screening of breast implant ruptures, beside the fact that it is an accessible tool.

\section{METHODS}

Search strategy: A search strategy was developed using the following terms: "Breast implant" "Rupture" and "Ultrasound" as text words and Medical Subject Headings (i.e., MeSH and EMTREE) and MEDLINE, EMBASE, Cochrane Library, Insight and Grey literature (Google Scholar and the British Library) was searched for studies published up to July 2020.

Participant or population: Women with mammary prostheses.

Intervention: Ultrasound of breasts.

Comparator: Breast surgery findings.

Study designs to be included: Observational, cross-sectional and cohort studies.

Eligibility criteria: Observational, crosssectional and cohort studies were included. The ultrasound diagnostic test was compared to the surgical findings as a reference standard, while applying the exclusion criteria for studies without complete verification by surgery.

Information sources: MEDLINE, EMBASE, Cochrane Library, Insight and Grey literature (Google Scholar and the British Library).

Main outcome(s): The present study performed a systematic review to identify the accuracy of ultrasonography to diagnose breast prosthesis rupture, studies in which the ultrasound diagnostic test was compared to a surgical finding as a reference standard.

Quality assessment / Risk of bias analysis: Methodological quality assessment of studies for diagnostic accuracy was performed according to criteria from the Quality Assessment of Diagnostic Accuracy Studies (QUADAS-2). These criteria assess the quality of included studies in terms of risk of bias and concerns regarding applicability over four domains.

Strategy of data synthesis: A $2 \times 2$ contingency table was constructed for each selected study. Rates were calculated as true positive (TP), false positive (FP), true negative (TN), and false negative (FN). When any cell containing " 0 " was present in the contingency table, 0.5 was added to all cells in all studies to facilitate calculations. Dichotomization of the contingency tables was performed as follows: (1) index test: cited findings suggesting intra or extracapsular rupture and (2) reference test: visible and touchable findings of intra or extracapsular rupture during surgery.

Subgroup analysis: For all studies, we calculated the true-positive rate (TPR; sensitivity), specificity, false-positive rate (FPR; 1 - specificity), and the diagnostic odds ratio (DOR). The DOR, which relates to different combinations of sensitivity and specificity, was calculated by (sensitivity/ (1-specificity))/((1- sensitivity)/specificity)) . A DOR indicated the assay had discriminative power. The DOR describes the odds of the positive test results in participants with disease compared to the odds of positive test results in those without disease. Bivariate analysis was used to calculate pooled estimates of sensitivity, specificity, and DOR with $95 \%$ confidence intervals (Cls) for summary estimates. To analyze the accuracy of ultrasound, the area under the curve (AUC) was calculated from the hierarchical summary receiver-operator curves (HSROC). AUC values $\geq 0.5,0.75,0.93$, and 0.97 were considered to represent fair, good, very good, and excellent accuracy, respectively.

Sensibility analysis: Heterogeneity of both sensitivity and specificity across the studies was tested using a X 2 analysis, with a $\times 2$ p-value $<0.05$ considered heterogeneous. As an alternative method to explore heterogeneity, the I 2 index was also utilized. The I 2 index presents the percentage of total variation across studies 
due to heterogeneity rather than chance; I 2 values of $75 \%$ or greater were considered substantial heterogeneity 15 . The metaanalysis was performed using Metadisc $₫$ 1.4 and Review Manager $\circledR$ (RevMan) version 5.3 software.

Language: English.

Country(ies) involved: Brazil.

Keywords: rupture, ultrasound, silicone prosthesis.

Contributions of each author:

Author 1 - Ana Cristina Macedo Lacerda Protocol/project development, data collection and management, data analysis, statistical analysis, manuscript writing/ editing.

Author 2 - Maria Laura Rodrigues Uggioni Protocol/project development, data collection and management, manuscript writing/editing.

Author 3 - George da Silva Carvalho Protocol/project development, data collection and management.

Author 4 - Carla Sasso Simon - Manuscript writing/editing.

Author 5 - Mateus Cruz - Manuscript writing/editing.

Author 6 - Fábio Silva - Manuscript writing/ editing.

Author 7 - Maria Inês da Rosa - Protocol/ project development, data collection and management, data analysis, statistical analysis, manuscript writing/editing. 